\title{
Study of measurements bias due to environmental and spatial discretization in long term thermal monitoring of structures by infrared thermography
}

\author{
by T.Toullier ${ }^{*, * *}$, J. Dumoulin ${ }^{*, * *}$, L. Mevel ${ }^{*, *}$ \\ * Ifsttar, COSYS-SIl, Route de Bouaye, F-44340, Bouguenais, France \\ ** Inria, 14S Team, Campus de Beaulieu, F-35042 Rennes, France
}

Abstract

The purpose of this study is to characterize the influence of environmental parameters for long-term in-situ structure monitoring as well as projections errors due to camera view and digitization. Measurements made on an instrumented test site have been made during the last 3 years, bringing an interesting dataset to exploit. The model used to convert the gathered data to temperature is firstly presented and discussed. Then, the effect of camera resectioning on infrared measurements is commented. Finally, the effect of the environmental parameters is studied and perspectives are proposed.

\section{Introduction}

Non-destructive thermal monitoring of transport infrastructure has become a major concern for next generation civil engineering structures. Therefore, new thermal monitoring solutions in-situ, low cost and robust are needed. If thermocouples or even optics fibers meet the expectation of thermal monitoring, their corresponding instrumentation may be complex with a risk of breaking during the construction (i.e. operation). At the opposite, infrared thermography seems to be simpler to deploy and offers a temperature measurement on multiple points at the same time. Even if the technological context of uncooled infrared cameras is evolving quickly, methods and processes for using them as an accurate and lowcost tool are still lacking.

The main challenge for using infrared cameras as a thermal monitoring tool is to convert the radiative fluxes received by the infrared camera to temperature. In fact, such task depends on spatial parameters, the environment and the observed object characteristics. Thanks to the improvement of acquisition and computation means, two instrumented multi-sensors tests sites have gathered an interesting amount of synchronized and coupled data over the last 3 years. This study aims to evaluate the impact of varying meteorological conditions and camera position adjustment on the infrared measurements. The experimental setups will be presented first. Then, the proposed conversion model for computing the temperature will be introduced. The camera resectioning and environmental variations error influences will be exposed and commented. Finally, outcomes are presented.

\section{Test site, related instrumentation and configuration}

\subsection{Test site \#1: instrumented road section}

Experiments have been conducted on an instrumented transport infrastructure section that can be heated. The studied section is monitored with a FLIR SC655 thermal infrared camera (640 X 480 LWIR FPA, pitch $17 \mu \mathrm{m}$, equipped with a $13.1 \mathrm{~mm}$ optical focal length). A weather station (VAISALA WXT 520), a net radiometer (CNR 4) and a pyranometer (SPLite2), are implemented on site to record meteorological and radiative heat fluxes conditions during IR image acquisition. Multi-sensor data acquisition system used is synchronised in time. Moreover, thermocouples have been inserted at surface level of the structure enabling us to compare infrared measurements with thermocouples ones. In the present study we consider one measurement campaign for a camera point of view and different meteorological conditions.

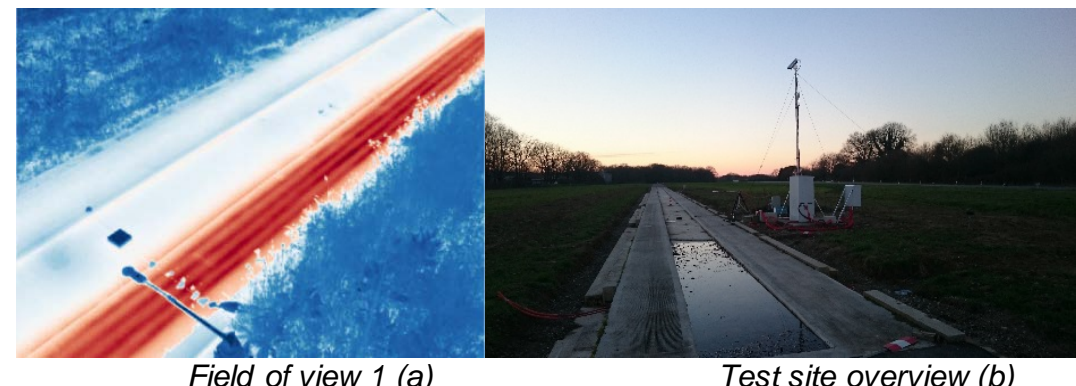

Fig. 1: IR camera spatial instrumentation on site: IR image for field of view considered (a) - test site overview (b) 


\subsection{Test site \#2: instrumented wood house}

Under the SenseCity project of IFSTTAR, a wood house has been built and instrumented for multiple years in Paris with well-known material properties and dimensions to have a real sized and in-situ mock-up. The house is monitored with a FLIR A65 thermal infrared camera (640 X 512 LWIR FPA, pitch $17 \mu \mathrm{m}$, equipped with a 13 mm optical focal length). A weather station (VAISALA WXT 520) and a sunshine pyranometer (SPN1) giving us the total and diffuse solar radiation. The same acquisition system as the first test site is used to get data synchronized in time. Again, the infrared camera is placed on a mast to get a large view of the scene.

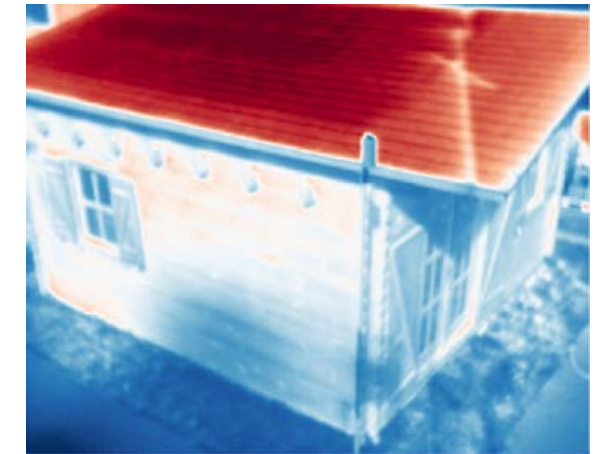

Image of the house in the infrared spectrum (a)

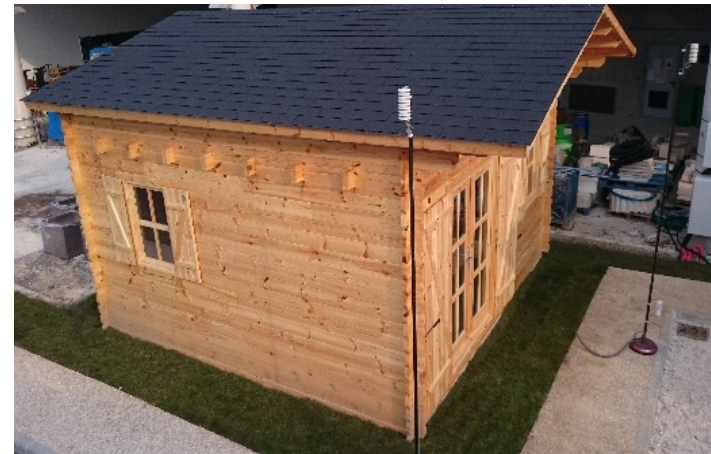

Image of the house in the visible spectrum (b)

Fig. 2: Wood house instrumentation: IR image (a) and test site overview (b)

\subsection{Camera resectioning}

Practical applications may need to focus on a particular region of interest on the image to retrieve the temperature at one precise location. Moreover, knowing the geometrical properties of the sensor-scene configuration on the measurement site is useful, in particular to get the view angle of the camera to the object. Retrieving the real world coordinates on the image is known as resectioning (see Fig. 3).

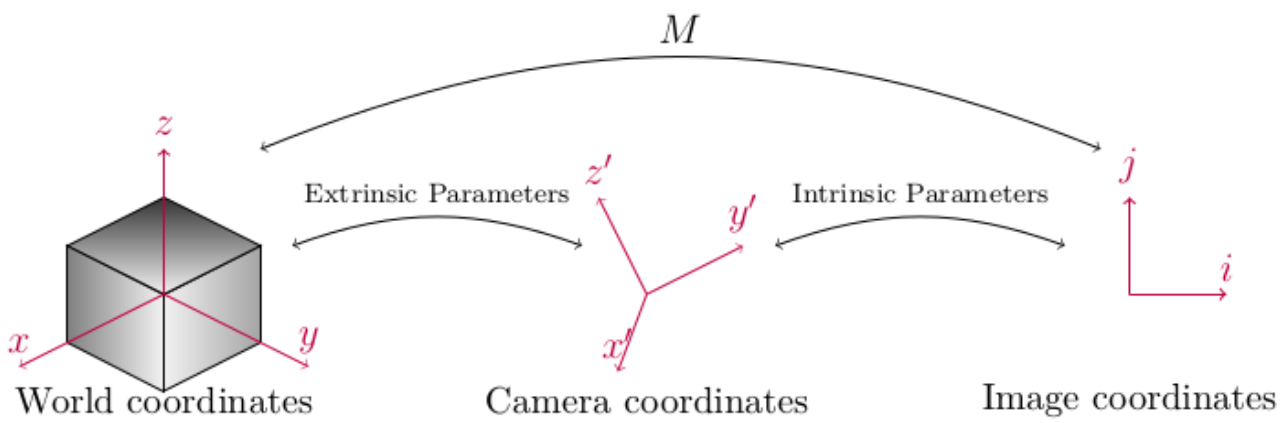

Fig. 3: Resectioning principle, from world coordinates to image coordinates

We define the calibration matrix $M$ such as for every couple $(x, X) \in \mathbb{R}^{2} \times \mathbb{R}^{3}$ of associated points between the image and the real world, we have:

$$
x=M X
$$

By using the pin-hole model, $M$ can be expressed in homogeneous coordinates as:

$$
M=\left(\begin{array}{ccc}
f & s_{k} & c_{x} \\
0 & \text { af } & c_{y} \\
0 & 0 & 1
\end{array}\right)\left(\begin{array}{cccc}
1 & 0 & 0 & 0 \\
0 & 1 & 0 & 0 \\
0 & 0 & 1 & 0
\end{array}\right)\left(\begin{array}{cc}
R_{3 \times 3} & 0_{3 \times 1} \\
0_{1 \times 3} & 1
\end{array}\right)\left(\begin{array}{cc}
I_{3} & T_{3 \times 1} \\
0_{1 \times 3} & 1
\end{array}\right)
$$

The system in Eq. (1) has 11 degrees of freedom, making necessary to have the knowledge of at least 6 correspondences points between the real world and the image. The Levenberg-Marquadt algorithm is finally used to get the calibration matrix estimation from those points [1], [2]. 


\section{Temperature computation based on a multi-sensor model}

The flux received at cameras' sensor depends on its surrounding environmental situation and also the thermooptic properties of the measurement scene [3], [4]. Furthermore, the camera is placed on a mast, leading to a non-constant spatial sampling of the monitored scene's surface. In such configuration, the radiation flux attenuated by the atmosphere depends on the position of the measurement point in the scene as illustrated in Fig. 4.

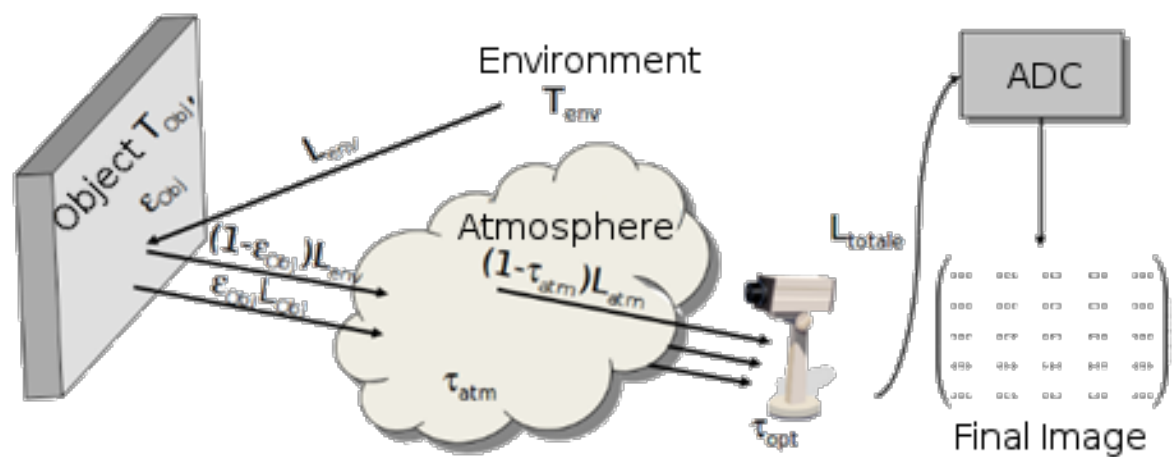

Fig. 4: Radiation interactions received by camera's sensors

The simplified radiometric equation is then used to retrieve the flux received by the camera at each pixel $L_{\text {total }}^{i, j}$, see Eq. (3). The weather station is used to compute the environmental quantities; the reflected, optics and atmospherics luminance values are computed by injecting some knowledge about the monitored objects, components of the infrared system and atmospheric conditions. In a first approach, we used literature's models [5] combined with inputs issued from in-situ solar and meteorological measurements for the reflected radiance contribution and for atmospheric transmission.

$$
L_{\text {total }}^{i, j}=\epsilon_{o b j} \tau_{a t m} \tau_{o p t} L_{o b j}^{i, j}+\left(1-\epsilon_{o b j}\right) \tau_{a t m} \tau_{o p t} L_{e n v}^{i, j}+\left(1-\tau_{a t m}\right) \tau_{o p t} L_{a t m}
$$

Finally, a RBF-function Eq. (4) is used to compute the temperature with a thermal calibration process; prior to the experiment [6].

$$
T_{o b j}^{i, j}=\frac{B}{\log \left(\frac{R}{L_{o b j}^{i, j}}-F\right)}
$$

Fig. 5 and Fig. 6 show the result of adjusting $R, B$ and $F$ values after such calibration.

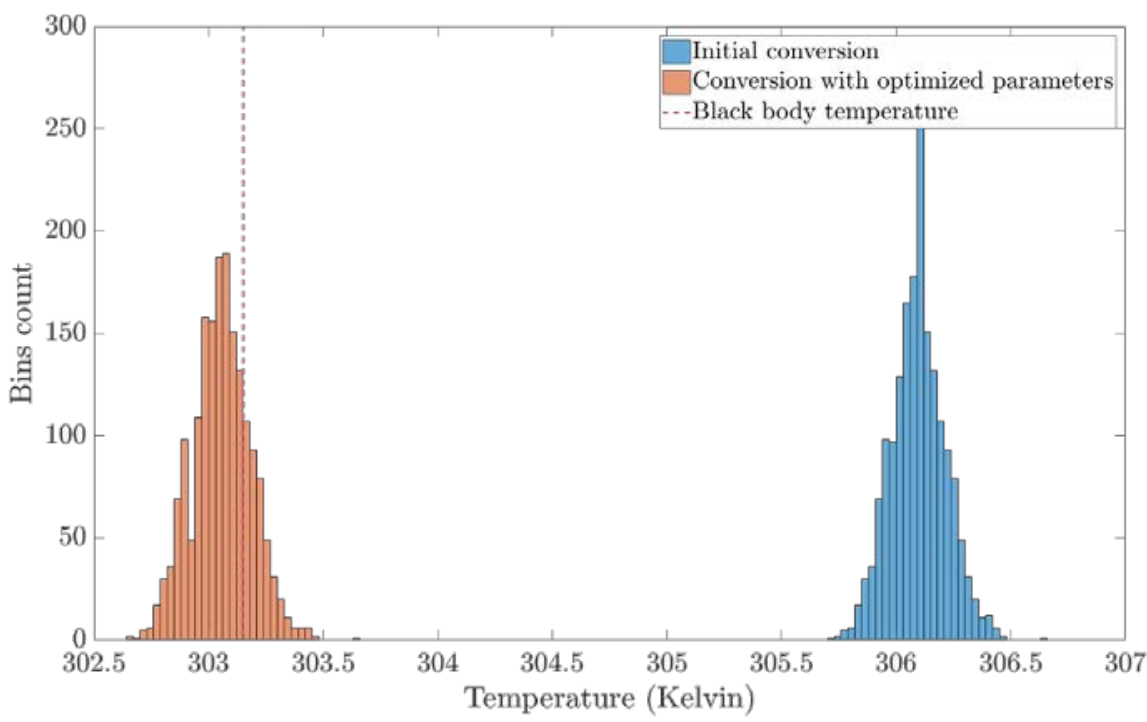

Fig. 5: Histograms of the calibration images, before and after parameters optimization for $T_{\text {calibration }}=303.15 \mathrm{~K}$. 


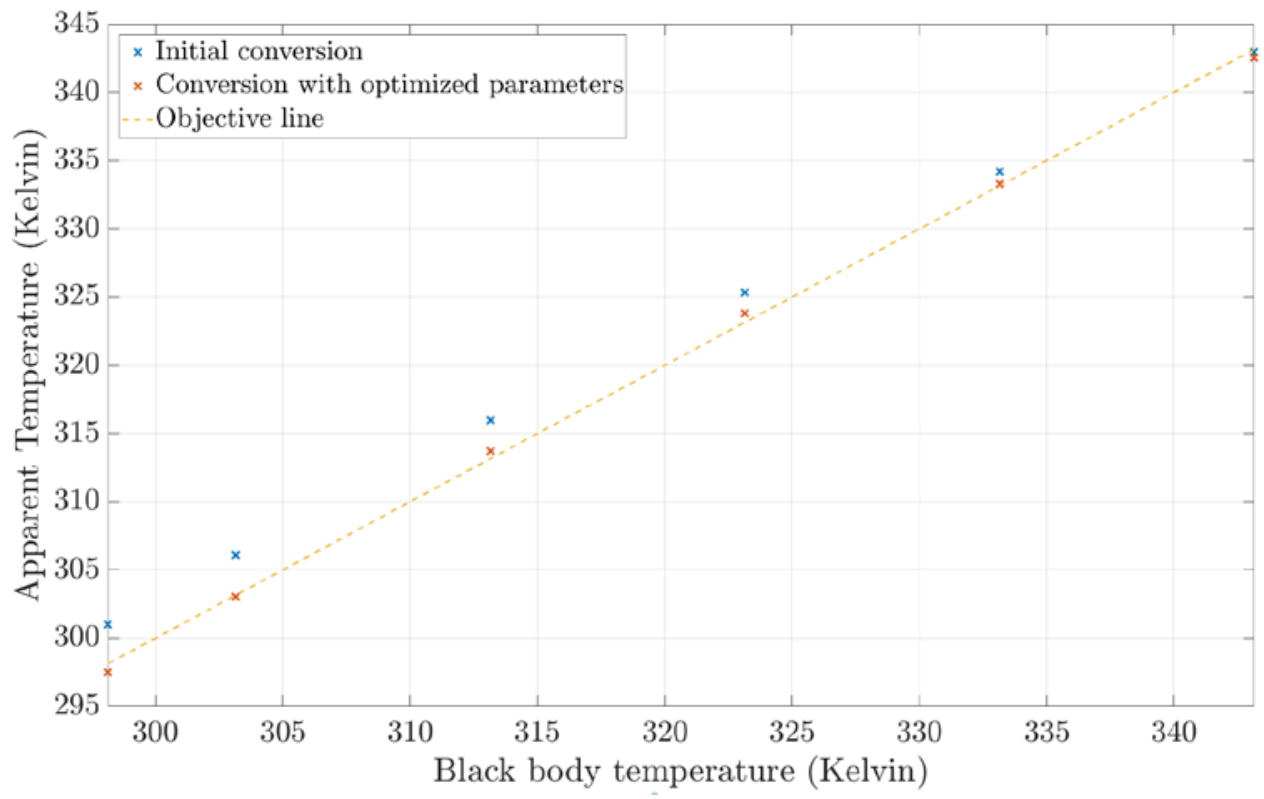

Fig. 6: Calibration optimization result

In the temperature interval considered up $3 \mathrm{~K}$ deviation was observed before recalibration.

\section{Spatial influence}

\subsection{Spatial resolution and angle of view}

Monitoring a civil engineering structure requires a large angle of view which forces users to use a mast to place the camera. This situation results in images with varying spatial resolution and important angle between the object and the camera (e.g. Fig. 1a). The underlying consequences are non-square pixels in the real-world and varying angles values along the same plane which has an impact on the emissivity values to be considered. One way to overcome this is to perform spatial calibration on images to be able to recover the region of interest. However, such operation induces a new sampling obtained by interpolation illustrated in Fig. 7.
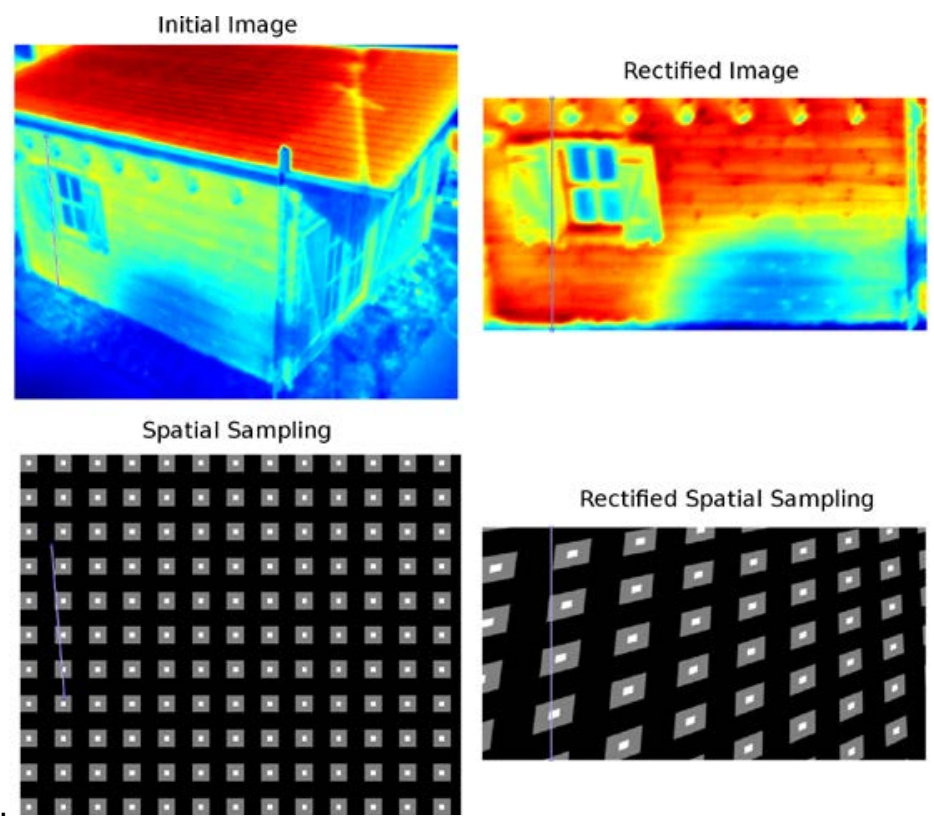

Rectified Spatial Sampling

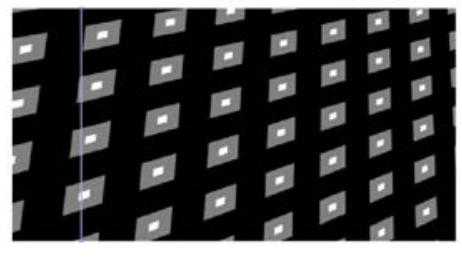

Fig. 7: Illustration of sampling issues after projection 
We can see in Fig. 8 the actual effect of the projection to the thermal profiles.

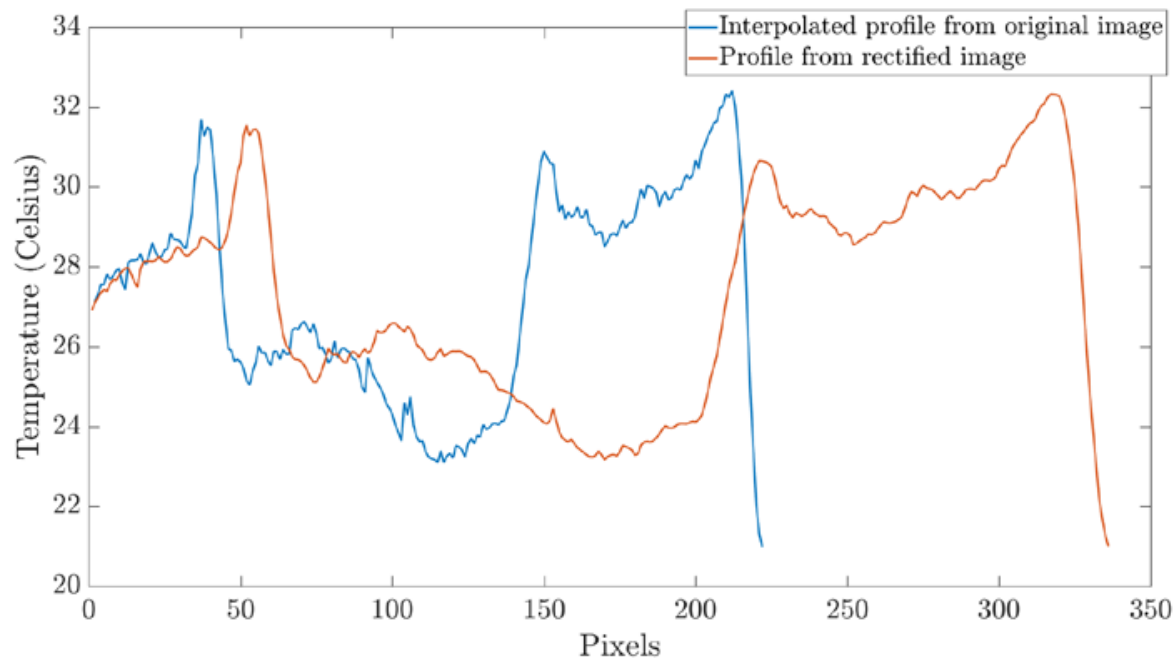

Fig. 8: Profiles from Fig. 7, before and after projection

The thermal profile is scaled up and smoothed due to the interpolation.

\section{Meteorological impact on temperature estimation error}

Various factors affect the temperature estimation based on infrared measurements [7]. We will review in this part the impact of the different errors made for the temperature estimation based on the model presented Eq. (3).

\subsection{Model sensitivity to emissivity}

Emissivity measurements have been made on the wood house (test site \#2) in order to get an emissivity map, represented in Fig. 9. Table 1 shows the associated emissivity measurements.

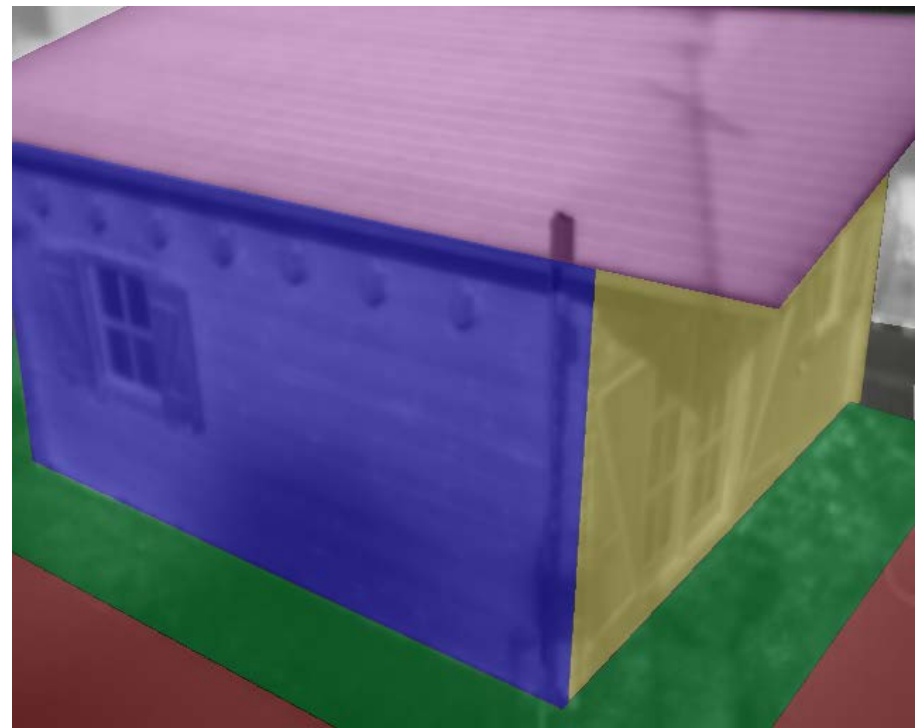

Fig. 9: Emissivity map for the wood house, colors represents one particular emissivity measurement

\begin{tabular}{c|c|c}
\multicolumn{1}{c}{ Material } & Color & Emissivity \\
\hline Wood wall (west) & Blue & $\mathbf{0 . 9 5}$ \\
\hline Wood wall (south) & Yellow & $\mathbf{0 . 9 0}$ \\
\hline Grass & Green & $\mathbf{0 . 9 5}$ \\
\hline Sidewalk & Red & $\mathbf{0 . 8 9}$ \\
\hline Tar & Grey & $\mathbf{0 . 9 5}$ \\
\hline Roof & Purple & $\mathbf{0 . 9 2}$ \\
\hline
\end{tabular}

Table 1: Emissivity measurements values in the $8 \mu \mathrm{m}-14 \mu \mathrm{m}$ band.

Fig. 10 shows the effect of adjusting the emissivity to a particular material. Unfortunately, the comparison can only be made relatively due to the lack of ground truth temperature measurements at the surface of the different materials.. 


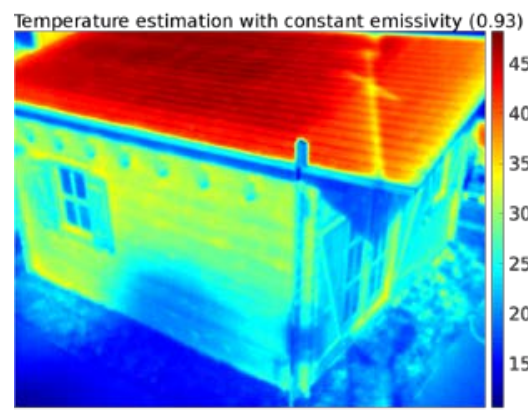

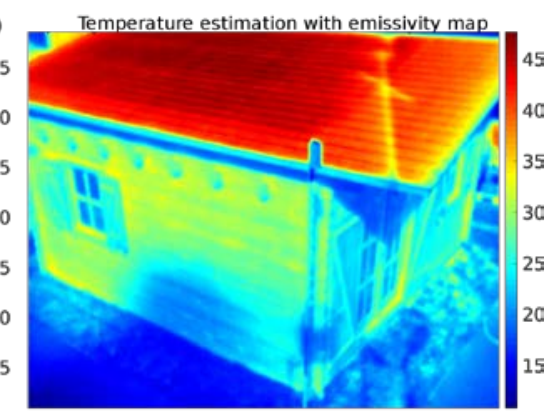

Absolute difference of the two images

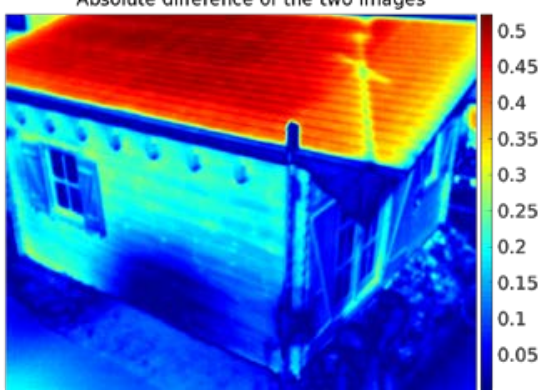

Fig. 10: Effect of adjusting the emissivity for different material compared to a constant emissivity

However, one can see that adjusting the emissivity will make the temperature estimation more accurate. In fact, in this example, we have chosen the best candidate for a constant emissivity value over all the image because the average emissivity value is 0.93 with a standard deviation of 0.025 (derived from Table 1). As a consequence, we can see that even when the best constant emissivity value is chosen, this is still leading to, at most, $0.5 \mathrm{~K}$ difference when using the emissivity map

\subsection{Model sensitivity to sky temperature}

The sky temperature $\left(T_{s k y}\right)$ will also have an impact on the final temperature estimation $\left(\widetilde{T}_{o b j}\right)$, as shown in Fig. 11 where the difference between the estimated sky temperature $\left(\widetilde{T}_{s k y}\right)$ is compared to the difference made on the estimated object temperature, for different values of $T_{o b j}$ and $T_{s k y}$. The $R, B$ and $F$ values used in the model to compute this figure are derived from a thermal calibration, as explained previously [8]. One can note that the those coefficients are only valid for a limited range of object temperature $(283.15 \mathrm{~K}-343.15 \mathrm{~K}$ in this case) and could expect some more important error out of this range.

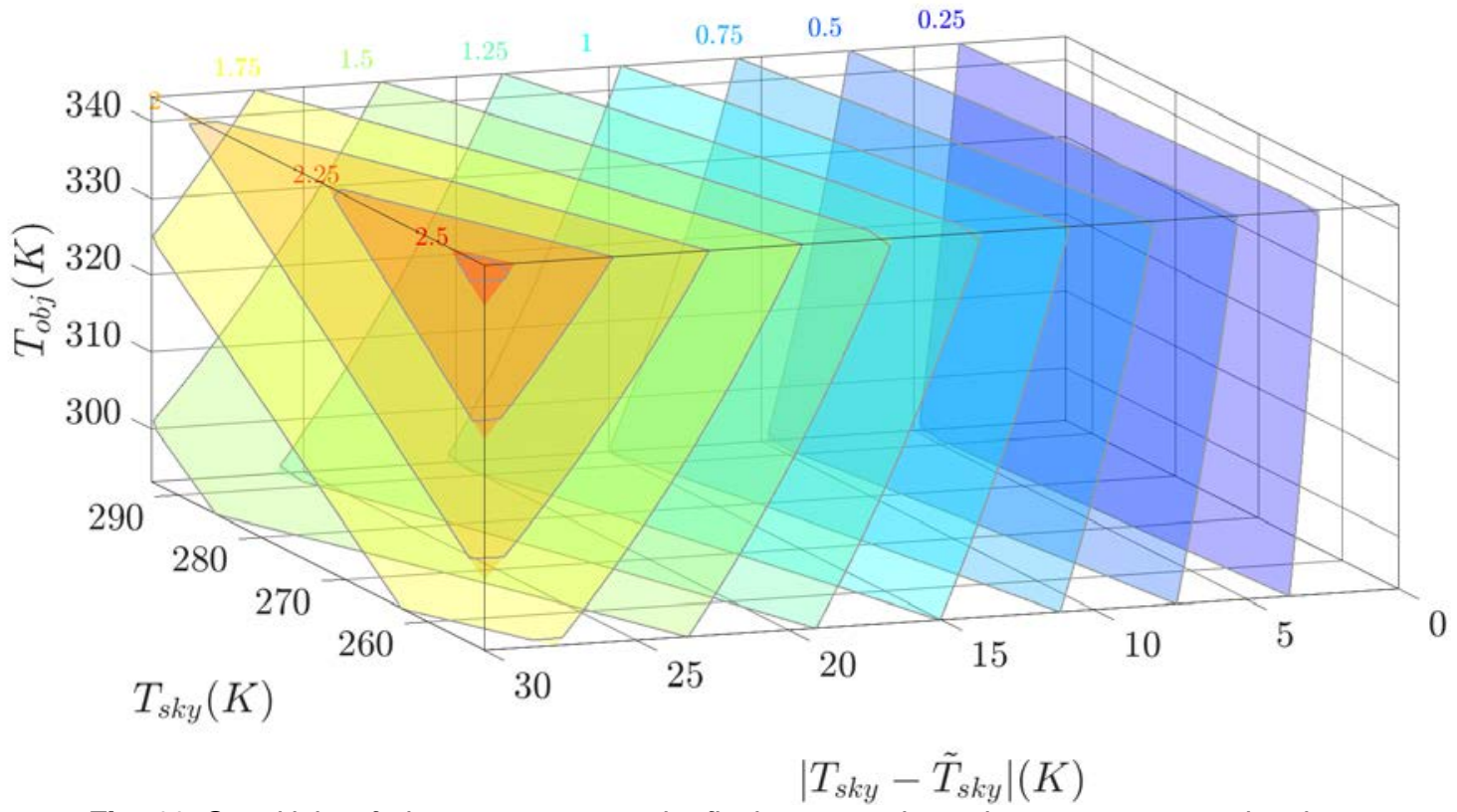

Fig. 11: Sensitivity of sky temperature to the final error made on the temperature estimation. Colors represents the isosurfaces of $\left|T_{o b j}-\tilde{T}_{o b j}\right|$ for different values of $T_{s k y}$ and $T_{o b j}$ and $\epsilon=0.95$ 
To overcome this, one can use the measurements made in-situ to get the sky temperature. In Fig. 12, the ground truth temperature obtained from thermocouples data is compared to the temperatures estimated with and without sky correction. In the case of the sky correction, the sky temperature is derived from a pyrgeometer data whereas a constant sky temperature is taken in the other case. The Fig. 12 (test site \#1) shows that taking into account the sky temperature from local measurements gives a better estimate of the object temperature. In this particular example, we compare the estimation of the temperature with and without the sky temperature correction to thermocouples measurements at the surface of the structure. The sky correction reduces in average the difference between the thermocouples measurements and the estimated temperature from infrared data.

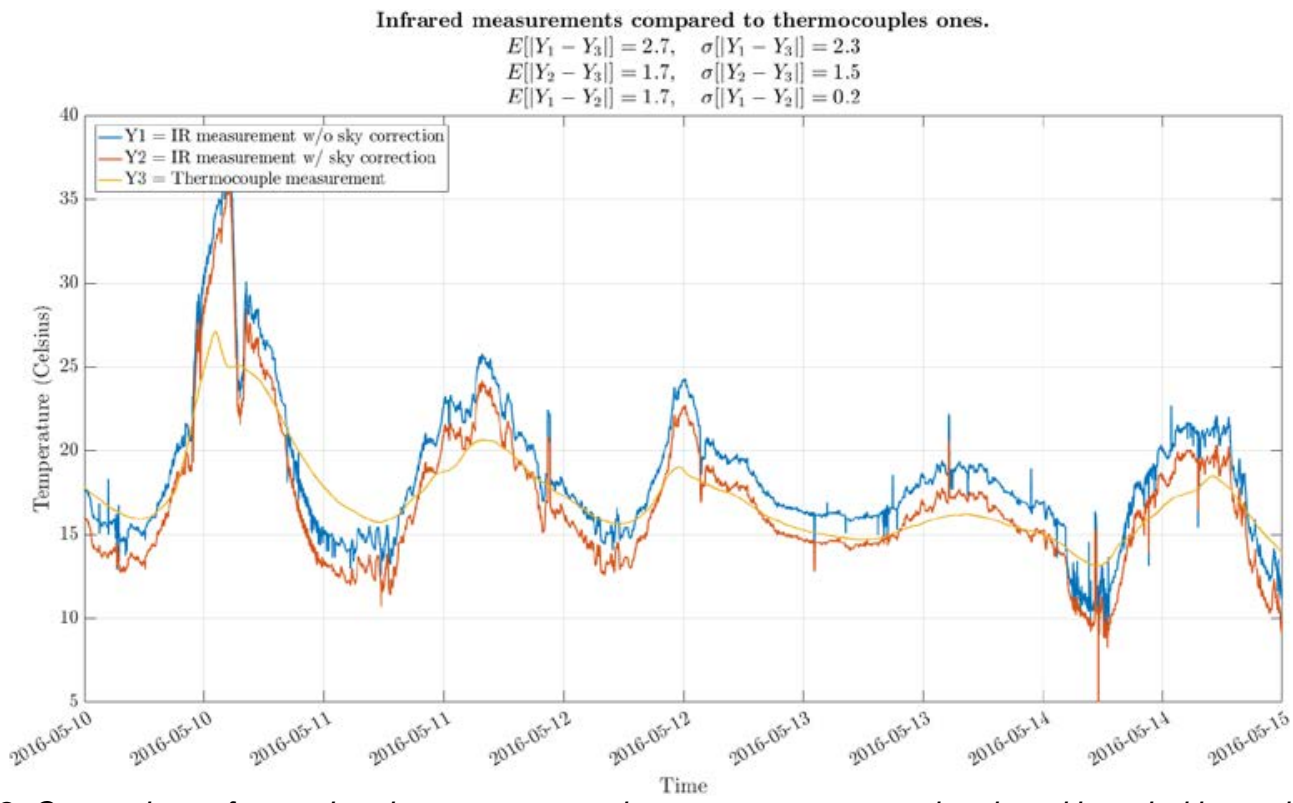

Fig. 12: Comparison of ground truth temperature values to temperature estimation with and without skycorrection.

Another possibility is to use correlations from air temperature to estimate the sky temperature. Such correlations can be found in the literature [9]. Fig. 13 shows the effect of Swinbanks correlation for two different periods: in december and august. One can see that the sky temperature will account for the final temperature estimate.

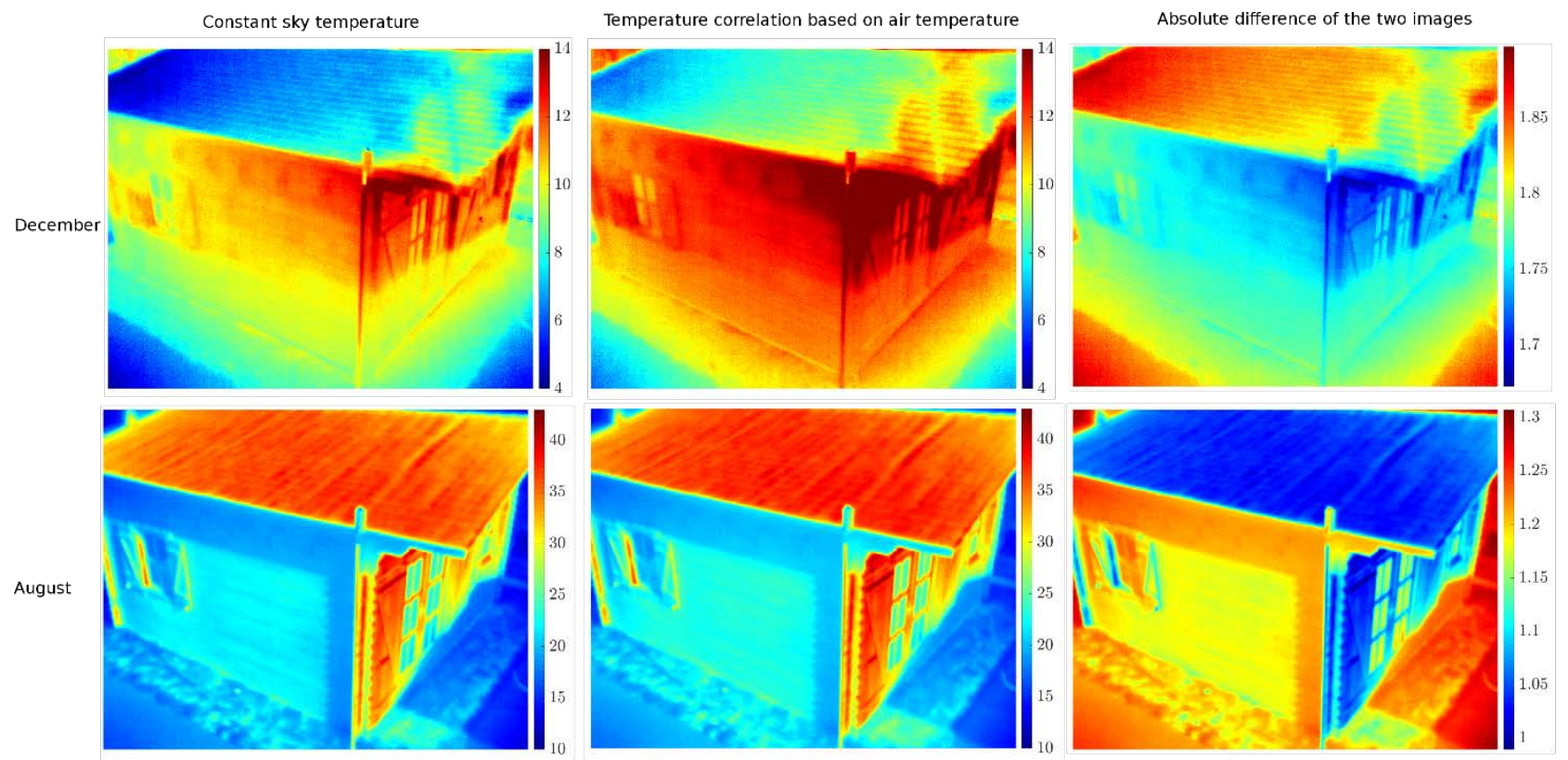

Fig. 13: Effect of using a correlation for the sky temperature based on the air temperature

Finally, the Fig. 14 illustrates the accumulation of errors made on the sky temperature and on emissivity to the estimation of temperature. 


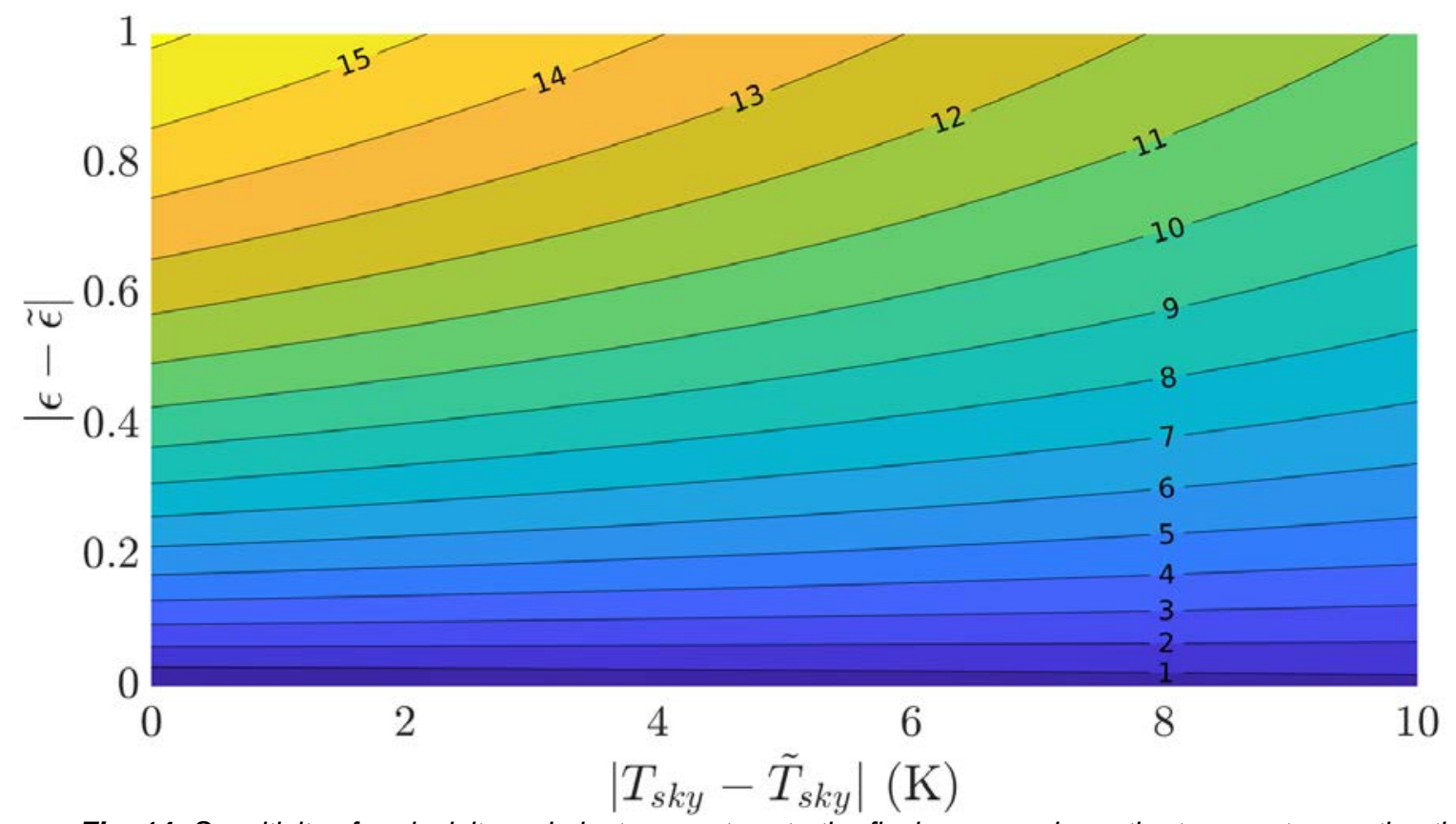

Fig. 14: Sensitivity of emissivity and sky temperature to the final error made on the temperature estimation. for $T_{\text {sky }}=273.15 \mathrm{~K}, T_{o b j}=293.15 \mathrm{~K}$ and $\epsilon=0.95$

\subsection{Wind and rain influence}

Rain and fog conditions will have an important impact on the flux received at camera's sensors. Fig. 15 illustrates the weather variation during a measurement campaign. Such variation will produce measurements that are difficult to exploit for accurate temperature estimation (see Fig. 16). Those may not lead to accurate temperature measurements. However, such effect could be compensated by using a black-body source and perform automatic thermal calibration in case of rain or using adequate radiative transmission model (for instance in case of fog [10]).

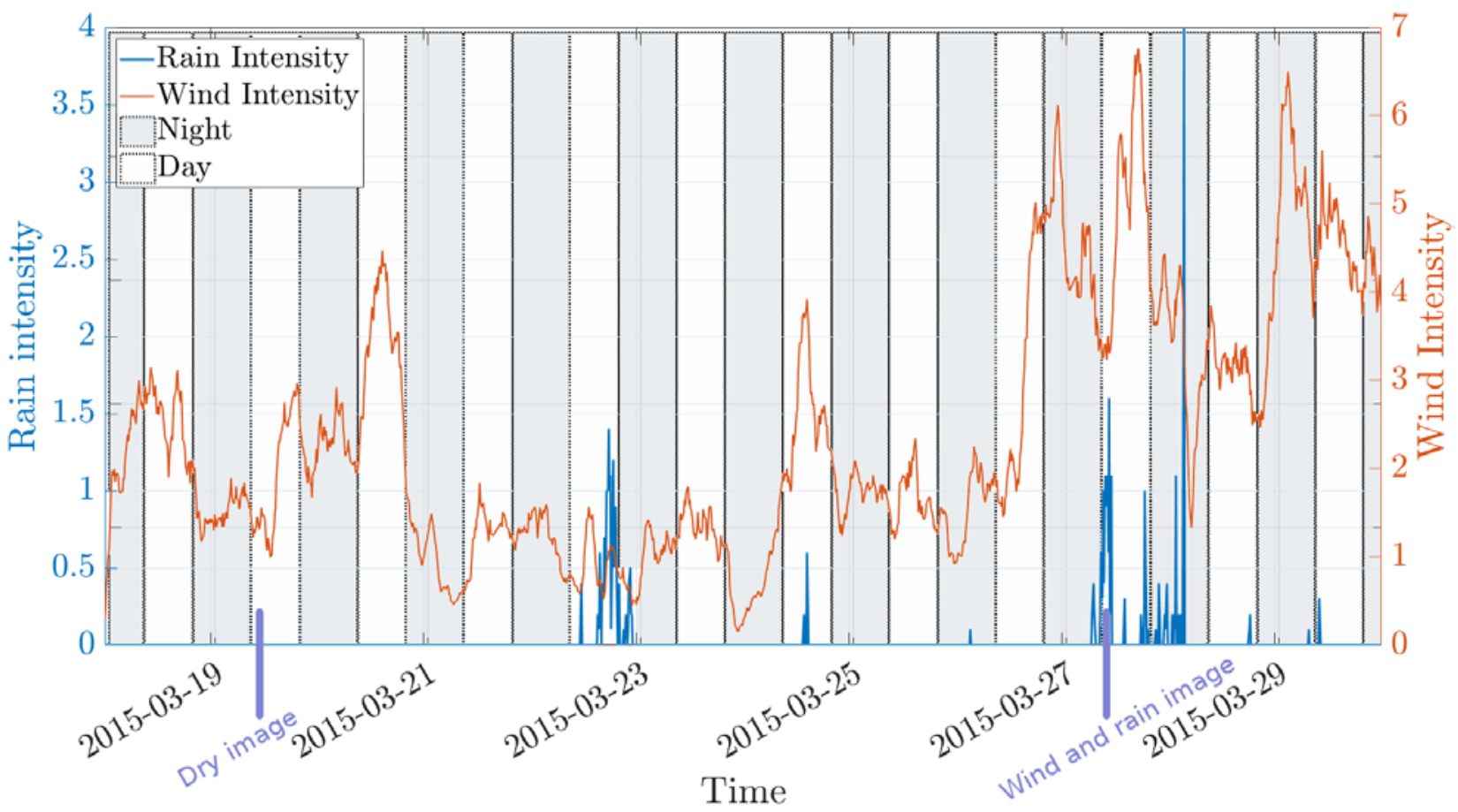

Fig. 15: Rain and wind measurements for test site \#2 


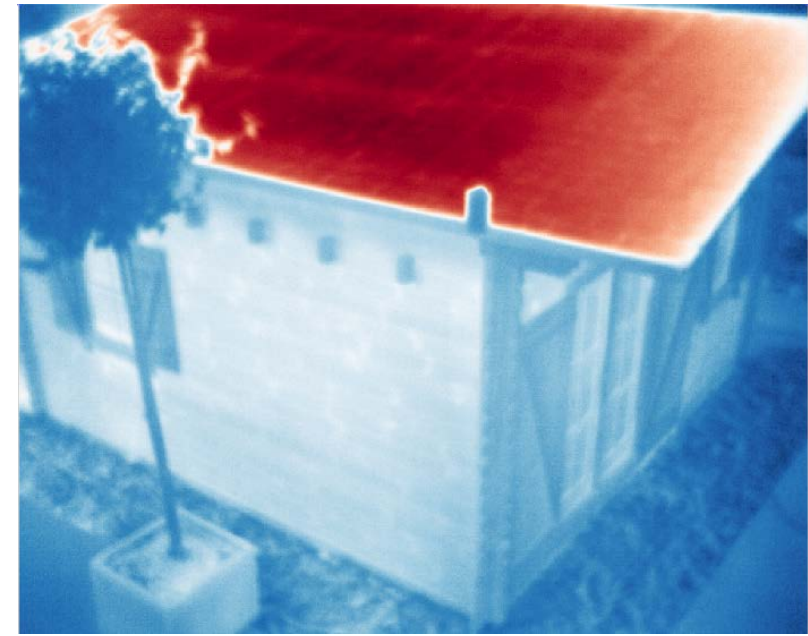

Dry situation (a)

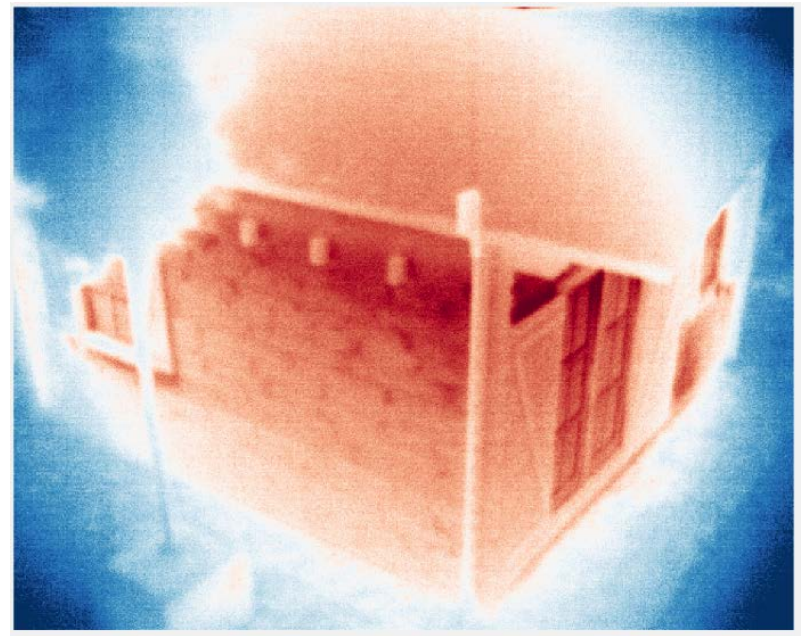

Raining with wind (b)

Fig. 16: IR image view. in dry environment (a), rain and wind situation (b)

\section{Conclusion}

A study of the different parameters that create bias in long term thermal monitoring of structures by infrared thermography has been conducted. To achieve in-situ accurate temperature estimation from those measurements, one need to take into account the environmental parameters, the geometry of the scene and the characteristics of the observed materials. Using local and coupled measurements with those infrared data enables the temperature estimation to be more accurate.

The spatial calibration presented in this study makes possible the extraction of region of interest. One other possibility is to use stereovision [11] to get a more accurate calibration and in particular to retrieve the depth in the image. Further investigation is required to apply computer vision algorithm for infrared images, since the infrared sensor's responses are different than in the visible spectrum. In any case, after resectioning, the resulting signal should be considered with caution since the sampling is not uniform. Moreover, the pixels of the image do not represent the same area in the real-world due to the perspective projection. Furthermore, one should also consider the "mixed pixel effect" [12] due to the spatial resolution for accurate infrared measurements. In fact, considering that one given pixel is homogenous can be discussed.

Finally, taking into account the actual sky temperature in our model as well as mapping the emissivity to the image materials enabled us to reduce the difference between the temperature estimation and the expected surface temperature. Estimating the sky temperature by a correlation gives satisfying results in a first approximation but should be tested for limit cases. However, wind and rain influence is not yet incorporated in our conversion model from digital levels to temperature. One of the prospects is to use the meteorological data coupled with a deep learning algorithm to compensate those effects. Moreover, one of our perspective is to use a Bayesian model to estimate conjointly the emissivity and the temperature.

\section{ACKNOWLEDGEMENTS}

Authors wish to thanks for their support: French Region Bretagne, THEMACS INGENIERIE for providing emissivity measurements on the test site \#2, and Sense-City Facility of Excellence of ANR's Future Investment Program.

\section{REFERENCES}

[1] E. K. P. Chong and S. H. Zak, An Introduction to Optimization, 4th Edition. Hoboken, New Jersey: Wiley-Blackwell, 2013.

[2] J. C. Pérez Muñoz, C. A. Ortiz Alarcón, A. . Osorio, C. . Mejía, and R. Medina, "Environmental Applications of Camera Images Calibrated by Means of the Levenberg-Marquardt Method," Comput. Geosci., vol. 51, pp. 74-82, Feb. 2013.

[3] J. R. Howell, R. Siegel, and M. P. Pinar, Thermal Radiation Heat Transfer., 5th ed. CRC Press, 2010.

[4] J. Dumoulin, A. Crinière, and R. Averty, "The Detection and Thermal Characterization of the Inner Structure of the 'Musmeci' Bridge Deck by Infrared Thermography Monitoring," J. Geophys. Eng., vol. 10, no. 6, p. 064003, Dec. 2013.

[5] E. P. Shettle and R. W. Fenn, "Models for the Aerosols of the Lower Atmosphere and the Effects of Humidity Variations on Their Optical Properties," Sep. 1979. 
[6] T. Toullier, J. Dumoulin, and L. Mevel, "Étude Comparative de Deux Approches, Thermocouples Intégrés et Thermographie Infrarouge, Pour La Surveillance Thermique d'une Infrastructure de Transport," in 25eme Congrès Français de Thermique, 2017.

[7] S. Van De Vijver, M. Steeman, N. Van Den Bossche, K. Carbonez, and A. Janssens, "The Influence of Environmental Parameters on the Thermographic Analysis of the Building Envelope," in 12th International Conference on Quantitative InfraRed Thermography (QIRT 2014), 2014.

[8] N. Le Touz, T. Toullier, and J. Dumoulin, "Infrared Thermography Applied to the Study of Heated and Solar Pavement: From Numerical Modeling to Small Scale Laboratory Experiments," in Thermosense: Thermal Infrared Applications XXXIX, 2017, vol. 10214, p. 1021413.

[9] L. Adelard, F. Pignolet-Tardan, T. Mara, P. Lauret, F. Garde, and H. Boyer, "Sky Temperature Modelisation and Applications in Building Simulation," Renew. Energy, vol. 15, pp. 418-430, Sep. 1998.

[10] J Dumoulin and V Boucher; "Infrared thermography system for transport infrastructures survey with inline local atmospheric parameter measurements and offline model for radiation attenuation evaluations," J. Appl. Remote Sens., 8(1), 084978 (2014). doi:10.1117/1.JRS.8.084978.

[11] R. I. Hartley and A. Zisserman, Multiple View Geometry in Computer Vision, Second. Cambridge University Press, ISBN: 0521540518, 2004.

[12] H. Jones and X. Sirault, "Scaling of Thermal Images at Different Spatial Resolution: The Mixed Pixel Problem," Agronomy, vol. 4, no. 3, pp. 380-396, Jul. 2014. 